\title{
Increased extracorporeal membrane oxygenation center case volume is associated with improved extracorporeal membrane oxygenation survival among pediatric patients
}

\author{
Tara Karamlou, MD, MSc, ${ }^{a}$ Mina Vafaeezadeh, MS, ${ }^{\mathrm{b}}$ Andrea M. Parrish, MS, ${ }^{\mathrm{b}}$ Gordon A. Cohen, MD, PhD, ${ }^{\mathrm{a}}$ \\ Karl F. Welke, MD, MS, ${ }^{\mathrm{b}}$ Lester Permut, MD, ${ }^{\mathrm{b}}$ and D. Michael McMullan, $\mathrm{MD}^{\mathrm{b}}$
}

Objective: We sought to examine the relationship between extracorporeal membrane oxygenation center case volume and survival in pediatric patients requiring extracorporeal membrane oxygenation support.

\begin{abstract}
Methods: Pediatric patients ( $\leq 20$ years) undergoing extracorporeal membrane oxygenation cannulation were identified using the Healthcare Cost and Utilization Project Kids' Inpatient Database for 2000 to 2009. Annual hospital extracorporeal membrane oxygenation volume tertiles were $<15$ patients/year (low volume), 15 to 30 patients/ year (medium volume), and $>30$ patients/year (high volume). Cases of extracorporeal membrane oxygenation were segregated by indication into cardiac and noncardiac groups. Cases of cardiac extracorporeal membrane oxygenation were mapped to Risk Adjustment for Congenital Heart Surgery categories to adjust for case complexity. Weighted multivariable logistic and linear regression models identified determinants of in-hospital mortality.
\end{abstract}

Results: Overall, 3867 cases of extracorporeal membrane oxygenation were identified, yielding a national estimate of $6333 \pm 495$ cases. Extracorporeal membrane oxygenation was used with nearly equivalent prevalence across volume tertiles for all Risk Adjustment for Congenital Heart Surgery categories, suggesting that patient selection for extracorporeal membrane oxygenation was fairly uniform. A higher annual extracorporeal membrane oxygenation volume tertile was associated with reduced in-hospital mortality $(P=.01)$ within nearly all Risk Adjustment for Congenital Heart Surgery categories. After adjustment for Risk Adjustment for Congenital Heart Surgery category and other patient variables, lower extracorporeal membrane oxygenation volume remained an important determinant of in-hospital death (odds ratio, 1.75; 95\% confidence interval, 1.03-2.94; $P=.03$ ).

Conclusions: Higher extracorporeal membrane oxygenation case volume is associated with improved hospital survival in pediatric cardiac extracorporeal membrane oxygenation patients. The results of this study may support the paradigm of regionalized centers of excellence for managing pediatric cardiac extracorporeal membrane oxygenation patients. (J Thorac Cardiovasc Surg 2013;145:470-5)

Earn CME credits at

http://cme.ctsnetjournals.org

In general, evidence supports a beneficial impact of institutional surgical case volume on in-hospital mortality and resource use in congenital cardiothoracic surgery. ${ }^{1-7}$ Despite the comparably high mortality and cost inherent in the provision of extracorporeal membrane oxygenation (ECMO)

\footnotetext{
From the Division of Pediatric Cardiac Surgery, ${ }^{a}$ University of California, San Francisco, Calif; and the Section of Pediatric Cardiothoracic Surgery, ${ }^{\mathrm{b}}$ University of Washington School of Medicine and Seattle Children's Hospital, Seattle, Wash. Disclosures: Authors have nothing to disclose with regard to commercial support. Read at the 38th Annual Meeting of The Western Thoracic Surgical Association, Maui, Hawaii, June 27-30, 2012.

Received for publication June 22, 2012; revisions received Sept 27, 2012; accepted for publication Nov 9, 2012; available ahead of print Dec 17, 2012.

Address for reprints: D. Michael McMullan, MD, Seattle Children's Hospital, Pediatric Cardiac Surgery, Mail Stop G-0035, 4800 Sand Point Way NE, Seattle, WA 98105 (E-mail: david.mcmullan@ seattlechildrens.org). 0022-5223/\$36.00

Copyright (c) 2013 by The American Association for Thoracic Surgery http://dx.doi.org/10.1016/j.jtcvs.2012.11.037
}

support, the relationship between center case volume and these outcomes within the context of pediatric ECMO have not been characterized. Investigation of institutional variables, including annual case volume, that modulate outcomes for pediatric ECMO has been limited for 3 main reasons: (1) ECMO is used in a diverse group of patients with divergent indications; (2) it is managed by different clinical providers (general surgeons vs cardiac surgeons) depending on institution precedent and diagnostic indication; and (3) the threshold for implementation, which may be an important determinant of outcome, is often dependent on individual provider and/or institutional bias. Despite these inherent limitations, however, it is probable that data from this heterogeneous population can be synthesized to estimate the impact of institutional factors. Enhanced understanding of whether institutional volume improves ECMO outcomes is important because the wisdom of extending regionalization initiatives to the provision of ECMO support requires evidence that such mandates would improve results. The current study evaluates whether annual institutional ECMO case volume influences in-hospital mortality among pediatric patients requiring ECMO. 


\section{Abbreviations and Acronyms \\ $\mathrm{ECMO}=$ extracorporeal membrane oxygenation \\ KID $=$ Kids' Inpatient Database \\ PHIS = Pediatric Health Information Systems \\ RACHS $=$ Risk Adjustment for Congenital Heart Surgery}

\section{METHODS}

\section{Patients and Data Set}

This study was designed as a retrospective cohort analysis using the Healthcare Cost and Utilization Project Kids' Inpatient Database (KID) (years 2000, 2003, 2006, and 2009). Pediatric patients (age $\leq 20$ years) who received ECMO support were identified using the International Classification of Diseases, Ninth Revision, Clinical Modification code 39.65. The KID is an administrative all-payer, pediatric, inpatient care database in the United States. For each year, the database contains data from between 2.5 to 3.4 million discharges for patients $\leq 20$ years of age, representing a stratified sampling of pediatric discharges from 2784 (2000), 3438 (2003), 3739 (2006), and 4121 (2009) U.S. community hospitals in 27 (2000), 36 (2003), 38 (2006), and 44 (2009) states. ${ }^{89}$ Systematic random sampling was used to select $10 \%$ of uncomplicated and $80 \%$ of complicated births. National estimates provided in the KID are calculated using discharge weights based upon the American Heart Association universe, and vary by year. The methodology for calculating the discharge weights and estimates for the KID are complex, but have been validated extensively. Detailed information regarding their calculation can be found at their Web site (http://www.hcup-us.ahrq.gov/db/nation/kid/KID_2009_Introduction.pdf). Briefly, discharge weights are calculated for KID data by stratifying the hospitals on the same variables that were used for creating the sample and then creating weights by stratum. The stratifying variables are geographic region, urban/rural location, teaching status, bed size, ownership, and children's hospital. The KID contains more than 100 clinical and nonclinical variables, and has been used by our group ${ }^{5,10,11}$ and others ${ }^{12,13}$ to study rare conditions such as congenital heart disease and procedures such as pediatric mechanical assist devices. Institutional review board approval was obtained for this study. All authors signed the KID Data Use Agreement as required.

\section{Hospitals and Cases}

Annual hospital ECMO volume was analyzed as a continuous variable, with subsequent segregation into ECMO volume tertiles ( $<15$ cases/year, low volume; $15-30$ cases/year, medium volume; $>30$ cases/year, high volume). Because the KID uses only $80 \%$ of complicated births, ECMO volumes were then multiplied by a factor of $1.25(0.80 \times 1.25=100)$. Sampling weights provided by the KID were used to calculate national estimates. Associated diagnosis codes were used to segregate ECMO cases by indication into primary cardiac or noncardiac. Cardiac cases were mapped to Risk Adjustment for Congenital Heart Surgery (RACHS) categories $^{14}$ to provide adjustment for case complexity. Timing of ECMO deployment was calculated based on the difference between the date of admission and the date on which ECMO occurred.

\section{Statistical Analysis}

Data are reported as medians with ranges or means with standard deviation depending on normality. Categorical variables were compared with the Rao-Scott $\chi^{2}$ test, and continuous variables were compared using a 2 -sided $t$ test. Statistical significance was set at a $P$ value $\leq .05$. Weighted multivariable logistic and linear regression models accounting for the stratified sampling scheme of the KID (PROC SURVEY) identified determinants of in-hospital mortality. A priori variables tested included patient factors such as age, gender, and ECMO indication, and hospital factors such as whether the hospital was a teaching hospital, bed size, and volume. Calendar year was included in all multivariable models to account for a possible era effect. Interactions were not evaluated. SAS software version 9.2 (SAS, Cary, NC) was used for all analyses.

\section{RESULTS \\ Patients and Hospitals}

We identified 3867 ECMO cases, yielding a national estimate of $6333 \pm 495$ ECMO cases. Cardiac ECMO cases predominated, with $593 \pm 97(10 \%)$ noncardiac cases and $5740 \pm 487(90 \%)$ cardiac cases overall. The majority of ECMO cases $(\mathrm{n}=4662 \pm 354 ; 2190 \pm 278$ [low] $+2471 \pm 327$ [medium]) occurred at either 145 low-volume hospitals or at 46 medium-volume hospitals, with only $1672 \pm 423$ cases performed at 16 high-volume hospitals. Patient characteristics were similar among the 3 volume groups, with the exception that high-volume ECMO centers performed a significantly higher proportion of cardiac ECMO cannulations (Table 1).

The mean time to ECMO deployment overall was $6.7 \pm 0.43$ days, and did not differ among the volume tertiles $(P=.74)$. Mean time to deployment was 6.5 days for low-volume centers, 6.9 days for medium-volume centers, and 6.9 days for high-volume centers.

Figure 1 demonstrates the case-complexity distribution of cardiac ECMO cases. Overall, 4954 of the 5740 cases $(86 \%)$ were able to be mapped to a RACHS category. Among cardiac ECMO cases, the majority of ECMO cases $(\mathrm{n}=3277)$ occurred in medium-complexity categories (RACHS-3 and -4 levels), which include patients who undergo surgical procedures such as arterial switch operation without ventricular septal defect, and complete repair of atrioventricular septal defect.

\section{In-Hospital Mortality}

Overall risk-unadjusted mortality was higher for cardiac ECMO compared with noncardiac ECMO $(2660 \pm 223$ $(46 \%)$ vs $230 \pm 43(39 \%)$, respectively; $P=.03)$. Figure 2 demonstrates risk-unadjusted in-hospital mortality segregated by ECMO volume tertile and indication. When segregated by volume tertile, low-ECMO volume hospitals had significantly higher in-hospital mortality compared with high-ECMO volume hospitals $(918 \pm 90(49 \%)$ vs $691 \pm 179(43 \%)$, respectively; $P=.015)$. However, when including medium-ECMO volume hospitals, which had intermediate mortality, there was still a strong trend suggesting lower volume was related to increased mortality, but statistical significance was not reached $(P=.08)$. There was no discernible relationship between ECMO case volume and mortality for noncardiac $\operatorname{ECMO}(P=.21)$.

Case-complexity distribution among ECMO volume tertiles was remarkably similar, suggesting that individual centers are using ECMO with equivalent frequency in most RACHS-complexity categories $(P=.84$; Figure $3, A)$. 
TABLE 1. Patient characteristics segregated by volume categories

\begin{tabular}{|c|c|c|c|c|}
\hline Variable & Low & Medium & High & $\begin{array}{c}P \\
\text { value }\end{array}$ \\
\hline $\begin{array}{l}\text { Age, year; mean } \\
\quad(\mathrm{SD})\end{array}$ & $1.9(4.7)$ & $1.5(4.1)$ & $1.3(3.6)$ & NS \\
\hline $\begin{array}{l}\text { Female, } \% \text {; NE } \\
\quad(\mathrm{SE})\end{array}$ & $43 ; 1233(103)$ & $44 ; 1351(182)$ & $44 ; 942(233)$ & NS \\
\hline Race, $\% *$ & & & & NS \\
\hline Caucasian & 46 & 50 & 53 & \\
\hline Asian & 21 & 18 & 15 & \\
\hline Black & 19 & 15 & 16 & \\
\hline Hispanic & 3 & 4 & 2 & \\
\hline Native American & 1 & 1 & $<1$ & \\
\hline Other & 10 & 12 & 14 & \\
\hline $\begin{array}{c}\text { Cardiac ECMO, } \\
\quad \% ; \mathrm{NE}(\mathrm{SE})\end{array}$ & $86 ; 1877$ (172) & $91 ; 2237(305)$ & $97 ; 1626(414)$ & .005 \\
\hline $\begin{array}{l}\text { Hospital length } \\
\text { of stay, days; } \\
\text { median (range) }\end{array}$ & $24(0-317)$ & $29(0-294)$ & $29(0-332)$ & \\
\hline
\end{tabular}

$S D$, Standard deviation; $N S$, not significant; $N E$, national estimate; $S E$, standard error; $E C M O$, extracorporeal membrane oxygenation. *Reporting of nonpercentages for categories that may have $<10$ discharges is not permitted.

However, within nearly all RACHS categories, there was a trend toward lower mortality in high-volume centers, although statistical significance was reached only within RACHS category 2 (Figure 3, B). For example, in the highest complexity, RACHS category 6 , which includes repair of hypoplastic left heart syndrome, the prevalence of death is $29 \%$ at high-volume centers compared with $43 \%$ at lowvolume centers.

\section{Independent Factors Associated With In-Hospital Mortality}

Multivariable patient factors associated with a reduction in the risk of in-hospital death are shown in Table 2. Patient

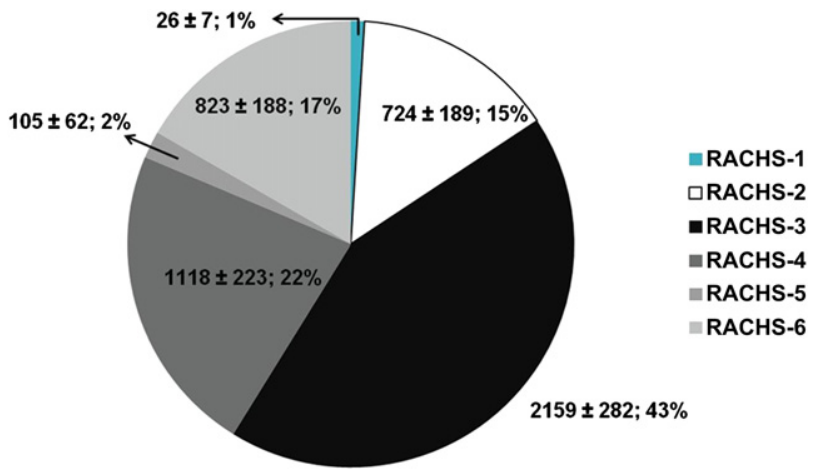

FIGURE 1. Cases of cardiac extracorporeal membrane oxygenation (ECMO) cases segregated by Risk Adjustment for Congenital Heart Surgery (RACHS)-1 category. As expected, the majority of cases fall within the RACHS-3 or -4 levels, which are the most commonly performed pediatric cardiac operations. The numbers accompanying each division of the pie chart represent the percent of cases within each category and the national estimates with their respective standard errors.

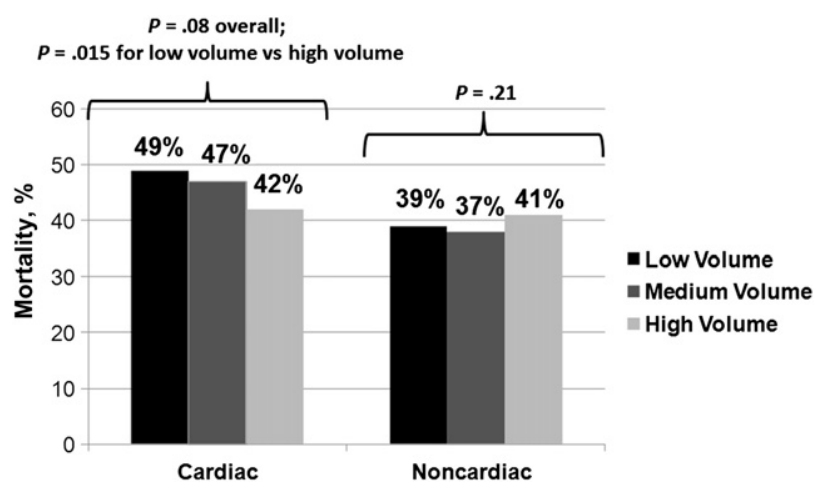

FIGURE 2. Risk-unadjusted in-hospital extracorporeal membrane oxygenation $(E C M O)$ mortality segregated by ECMO volume group tertile and indication. Note that mortality for cardiac ECMO is higher than for noncardiac ECMO. Percentages at the top indicate the mortality within each ECMO volume group. Low-volume hospitals have significantly higher mortality among cases of cardiac ECMO compared with highvolume hospitals $(P=.015)$. For noncardiac ECMO, the same trend applies, but statistical significance was not reached.

variables associated with improved survival included older age and assignment to RACHS category 3 . After adjustment for patient factors and case complexity, high-volume ECMO centers had significantly lower in-hospital mortality compared with low-volume centers, with a reduction of approximately $50 \%$ in the odds of death. Interestingly, no independent influence of time was observed, indicating a lack of era-specific improvement in ECMO mortality.

\section{DISCUSSION}

We have shown, using a large national data set, that higher annual ECMO volume is an important determinant of improved in-hospital survival for pediatric patients requiring ECMO support. We are aware of only 1 other study that investigated the influence of hospital volume on ECMO survival. ${ }^{15}$ Jen and Shew ${ }^{15}$ used the California Patient Discharge Database to study 188 children (age 1 month-18 years) undergoing ECMO from 1999 to 2006. They found a 1.34 times greater likelihood of survival with each standard deviation increase above a mean of 14 ECMO cases per year. Increasing hospital volume, as pointed out by previous studies, ${ }^{1-5,10,11}$ is likely a surrogate for other hospital characteristics (monetary resources, comprehensive array of diagnostic tools and personnel, and an experienced ECMO team) that contribute to improved outcomes. However, these variables are more difficult to measure accurately, lack objectivity, and are not captured with reasonable fidelity in available national data sets.

We also found an important dichotomy based on ECMO indication, in that mortality benefit of high-volume centers appears to be restricted to pediatric patients requiring cardiac ECMO. This dichotomy may have important implications regarding potential ECMO and pediatric 


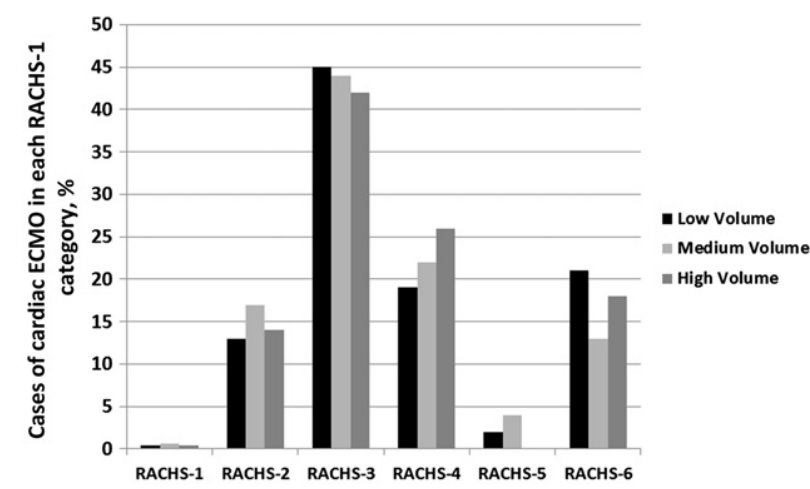

A

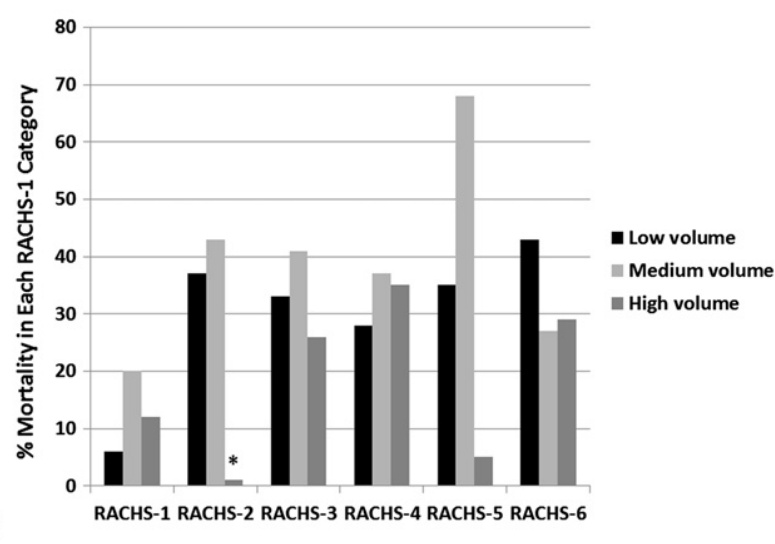

FIGURE 3. A, Colored histograms depict the percent of annual cardiac cases of extracorporeal membrane oxygenation (ECMO) within each Risk Adjustment for Congenital Heart Surgery (RACHS)-1 category segregated by center volume tertile. There were no differences among center volume tertiles regarding the prevalence of ECMO within each complexity category, indicating that centers use cardiac ECMO similarly across the case complexity spectrum $(P=.84)$. B, Colored histograms depict the percent mortality for cases of cardiac ECMO within each RACHS-1 category. Note that for nearly every category, there was a trend toward lower mortality for high-volume ECMO centers compared with low volume ECMO centers, although statistical significance was reached only in cases in the RACHS- 2 category. For RACHS-6, which includes repair of hypoplastic left heart syndrome, mortality in low-volume centers was $43 \%$ compared with $29 \%$ in high volume centers. Note that the wide variation in the RACHS- 5 category is a result of the paucity of cases mapped to this category $(\mathrm{n}=64) . P<.001$.

cardiac surgery regionalization initiatives. ${ }^{1,7,16}$ Although previous data regarding the impact of volume on outcomes in pediatric cardiac surgery are not conclusive and often inconsistent, ${ }^{3-5,17,18}$ and we have argued previously ${ }^{4,17,19-21}$ that volume as an isolated variable is a rather poor discriminatory metric, there is a growing body of evidence that mortality following pediatric cardiac surgery is inversely proportional to both annual institutional and surgeon volume. ${ }^{1-6,13}$ Based on these data, several authors have proposed that high-complexity pediatric cardiac surgery should be performed at highvolume centers to improve outcomes. ${ }^{2-7}$ Although we
TABLE 2. Multivariable model for in-hospital mortality

\begin{tabular}{|c|c|c|c|}
\hline Variable & OR & $95 \%$ CI & $P$ value \\
\hline Older age & 1.03 & $1.01-1.05$ & .005 \\
\hline \multicolumn{4}{|l|}{ Year } \\
\hline 2000 & \multicolumn{2}{|c|}{ Reference } & \\
\hline 2003 & 1.16 & $0.85-1.58$ & .35 \\
\hline 2006 & 1.26 & $0.94-1.68$ & .12 \\
\hline 2009 & 1.10 & $0.83-1.46$ & .52 \\
\hline \multicolumn{4}{|l|}{ Volume group } \\
\hline Low volume & \multicolumn{2}{|c|}{ Reference } & \\
\hline Medium volume & 0.78 & $0.59-1.01$ & .06 \\
\hline High volume & 0.51 & $0.30-0.87$ & .01 \\
\hline \multicolumn{4}{|l|}{ RACHS category } \\
\hline RACHS 1 & 0.67 & $0.23-1.92$ & .46 \\
\hline RACHS 2 & 0.82 & $0.63-1.07$ & .14 \\
\hline RACHS 3 & 0.78 & $0.64-0.96$ & .02 \\
\hline RACHS 4 & 0.94 & $0.75-1.19$ & .63 \\
\hline RACHS 5 & 1.32 & $0.77-2.23$ & .32 \\
\hline RACHS 6 & \multicolumn{2}{|c|}{ Reference } & \\
\hline
\end{tabular}

Model fit: $\mathrm{C}$ statistic $=0.6 . O R$, Odds ratio; $C I$, confidence interval; $R A C H S$, Risk Adjustment for Congenital Heart Surgery.

found that ECMO support was used with equivalent frequency in all RACHS categories across the spectrum of volume tertiles, high-volume centers performed better than low-volume centers in nearly all complexity categories. The results of this study suggest, therefore, that the provision of cardiac ECMO support should be considered when planning broader regionalization initiatives for complex pediatric cardiac surgery. Regionalization of care, however, must be balanced in terms of limiting access to care and requiring the transfer of critically ill patients, which may lead to adverse outcomes. More important, although we cannot explain directly the reason that highvolume centers had dramatically reduced mortality among ECMO cases in RACHS category 2, this finding does underscore the notion that even conceptually simple cases can have unexpected outcomes, and that in these cases, ECMO can be a life-saving therapy. Further studies are necessary to determine the feasibility, impact, and ideal structural design of regionalization in congenital cardiac surgery.

There are multiple reasons why high-volume centers may have improved results with cardiac ECMO. Earlier timing of ECMO deployment has been recommended by others. ${ }^{22,23}$ Preemptive use of ECMO may lead to more favorable clinical patient status, before the onset of multisystem organ failure, and could also lead to more favorable circumstances surrounding cannulation. We did not find any difference in ECMO deployment among volume tertiles, although we were constrained by the necessity to use date of admission as time 0 because our data set was constructed by using ECMO as the index procedure. In some cases, ECMO deployment could, conceivably, be better calculated based on an alternate reference point (ie, the time from another index procedure). Unfortunately, we could not 
ascertain the patient's clinical condition at the time of ECMO deployment from the current data set, but differences in illness severity certainly may have contributed to our results.

We were surprised by the paucity of noncardiac ECMO cases found in the KID. Based on extracorporeal life support organization registry data (2000-2009), there were approximately 6181 cardiac ECMO cases and 9597 noncardiac ECMO cases in a contemporaneous period. ${ }^{24}$ The basis for this discrepancy is poorly understood, but the difference is consistent with data reported in a previous study ${ }^{15}$ that also used an administrative data set in which respiratory failure accounted for only $12 \%$ of the total ECMO cases. Cardiac case allocation in our current study was assigned initially by identification of those cases that mapped to discrete RACHS categories (indicating the presence of a cardiac surgical procedure during the index hospitalization), and was verified subsequently by cross-checking individual diagnosis codes for a $30 \%$ sample of mapped cases and all nonmapped cases. It is possible that noncardiac ECMO cases are not captured with equal fidelity in administrative data sets, considering that such data sets are designed for claims collection and billing and therefore may be biased in favor of recording cases in which a cardiac surgical procedure was performed.

\section{Study Limitations}

Our data were obtained retrospectively from a large administrative data set. Missing data or miscoded data are potential sources of bias that cannot be excluded. However, the large sample size used in our study minimizes the impact of individual coding errors. The KID is also an incomplete (80\%) sample of complicated hospital discharges. However, its sampling algorithm used has been validated extensively and shown to provide accurate and robust national estimates in categories for which sufficient data exist. We attempted to account for the $80 \%$ sample by multiplying annual ECMO case volumes by 1.25 . Case-complexity assignment in this study used the RACHS methodology. Although more recent iterations (Society of Thoracic Surgeons-European Association of Cardiothoracic Surgeons Mortality category, Aristotle score $)^{25,26}$ for determining risk adjustment are available, RACHS was chosen for our analysis because it was designed and validated for use with administrative data. ${ }^{14}$ In addition, our group has used this mapping algorithm with both the KID and the Nationwide Inpatient Sample in several previously published studies. ${ }^{1,4,5,10,11,17}$ The categorization of ECMO volume tertiles was somewhat arbitrary. However, volume tertiles were optimized from the raw data to be analytically pragmatic, given the polarity of volume distribution with very few centers performing high ECMO case volumes.

It is plausible that specific improvements may have been distributed unequally across volume categories (ie, higher volume centers may have adopted newer technologies earlier). Although we cannot exclude the possibility of such an era effect, we did use calendar year to account for changes in management and technology during the study period. Last, we were not able to determine the duration of ECMO or the clinical circumstances surrounding cannulation. These issues are critical components of outcome according to recent reports ${ }^{22,23}$ and may have influenced our results.

Although other administrative databases exist, such as the Pediatric Health Information Systems (PHIS) database, these databases have limited participation from selected hospitals that could increase informative bias and limit our ability to capture low-volume centers. The PHIS database currently has only 41 participating hospitals across the United States, with essentially no participants from the central United States. The PHIS database could be ideal for analyzing longitudinal outcomes associated with ECMO use, including readmission and reintervention.

\section{CONCLUSIONS}

Higher annual center ECMO case volume is associated with improved hospital survival in pediatric cardiac ECMO patients. Annual ECMO case volume is an independent predictor of cardiac ECMO survival even when comparing patients of similar clinical complexity. Regionalization of care, in which the majority of cardiac ECMO support is concentrated in large, high-volume centers, could be considered a means of reducing resource use and improving in-hospital survival.

\section{References}

1. Karamlou T, Diggs BS, Person T, Ungerleider RM, Welke KF. National practice patterns for management of adult congenital heart disease: operation by congenital surgeons decreases in-hospital death. Circulation. 2008;118: 2345-52.

2. Pasquali SK, Li JS, Burstein DS, Sheng S, O’Brien SM, Jacobs ML, et al. Association of center volume with mortality and complications in pediatric heart surgery. Pediatrics. 2012;129:370-6.

3. Karamlou T, McCrindle BW, Blackstone EH, Caldarone CA, Cai S, Ashburn DA, et al. Lesion specific outcomes are related predominantly to patient management factors rather than institutional volume: a Congenital Heart Surgeons Society study. J Thorac Cardiovasc Surg. 2010;139:569-77.

4. Welke KF, Diggs BS, Karamlou T, Ungerleider RM. The relationship between hospital surgical case volumes and mortality rates in pediatric cardiac surgery: a national sample, 1988-2005. Ann Thorac Surg. 2008;86:889-96.

5. Welke KF, Diggs BS, Karamlou T, Ungerleider RM. Comparison of pediatric cardiac surgical mortality rates from national administrative data to contemporary clinical standards. Ann Thorac Surg. 2009;87:216-22.

6. Hornik CP, He X, Jacobs JP, Li JS, Jaquiss RD, Jacobs ML, et al. relative impact of surgeon and center volume on early mortality after the Norwood operation. Ann Thorac Surg. 2012;93:1992-7.

7. Chang RK, Klitzner TS. Can regionalization decrease the number of deaths for children who undergo cardiac surgery? A theoretical analysis. Pediatrics. 2002;109:173-81.

8. Healthcare Cost and Utilization Project. Overview of the KIDs' Inpatient Database. Available at: http://www.hcup-us.ahrq.gov/kidoverview.jsp. Accessed June 4, 2012.

9. Healthcare Cost and Utilization Project. Sampling of discharges: introduction to the HCUP-KIDS' Inpatient Database (KID), 2000, 2003, 2006, 2009. Available at: http://www.hcup-us.ahrq.gov/db/nation/kid/kid_2000_introduction.jsp. Accessed June 4, 2012. 
10. Caddell K, Diggs BS, de la Cruz, Walker W, Ehieli E, Karamlou T, et al. Common ground: measuring intrainstitutional mortality among pediatric surgical procedures. Surgery. 2011;149:7-14.

11. Karamlou T, Diggs BS, Ungerleider RM, Welke KF. Adults or big kids: what is the ideal clinical management for patients with grown-up congenital heart disease? Ann Thorac Surg. 2010;90:573-9.

12. Morales DLS, Zafar F, Rossano JW, Salazar JD, Jefferies JL, Graves DE, et al. Use of ventricular assist devices in children across the United States: analysis of 7.5 million pediatric hospitalizations. Ann Thorac Surg. 2010;90:1313-9.

13. Hirsch JC, Gurney JG, Donohue JE, Gebremariam A, Bove EL, Ohye RG. Hospital mortality for Norwood and arterial switch as a function of institutional volume. Pediatr Cardiol. 2008;29:713-7.

14. Jenkins KJ, Gauvreau K. Center-specific differences in mortality: preliminary analyses using the risk adjustment in congenital heart surgery (RACHS-1) method. J Thorac Cardiovasc Surg. 2002;124:97-104.

15. Jen HC, Shew SB. Hospital readmissions and survival after nonneonatal pediatric ECMO. Pediatrics. 2010;125:1217-23.

16. Chang RK, Klitzner TS. Resources, use, and regionalization of pediatric cardiac services. Curr Opin Cardiol. 2003;18:98-101.

17. Welke KF, Karamlou T, Ungerleider RM, Diggs BS. Mortality is not a valid indicator of quality differences between pediatric cardiac surgical programs. Ann Thorac Surg. 2010;89:139-44.

18. Bazzani LG, Marcin JP. Case volume and mortality in pediatric cardiac surgery patients in California, 1998-2003. Circulation. 2007;115:2652-9.
19. Welke KF, Diggs BS, Karamlou T. Chance, bias, and confounding: threats to valid measurement of quality in the context of pediatric cardiac surgery. Semin Thorac Cardiovasc Surg Pediatr Card Surg Annu. 2010;13:79-83.

20. Welke KF, Diggs BS, Karamlou T, Ungerleider RM. Measurement of quality in pediatric cardiac surgery: understanding the threats to validity. ASAIO J. 2008; 54:447-50.

21. Petrou S, Bischof M, Bennett C, Elbourne D, Field D, McNally H. Cost-effectiveness of neonatal extracorporeal membrane oxygenation based on 7-year results from the United Kingdom Collaborative ECMO Trial. Pediatrics. 2006;117: 1640-8.

22. Sherwin ED, Gauvreau K, Scheurer MA, Rycus PT, Salvin JW, Almodovar MC, et al. Extracorporeal membrane oxygenation after stage 1 palliation for hypoplastic left heart syndrome. J Thorac Cardiovasc Surg. 2012;144:1337-43.

23. Itoh H, Ichiba S, Ujike Y, Kasahara S, Arai S, Sano S. Extracorporeal membrane oxygenation following pediatric cardiac surgery: development and outcomes from a single-center experience. Perfusion. 2012;27:225-9.

24. Extracorporeal Life Support Organization. ELSO registry cases for 2000-2009. Available at: http://www.elso.med.umich.edu/Registry.html. Accessed June 19, 2012.

25. Pasquali SK, Xia HE, Jacobs JP, Jacobs ML, O'Brien SM, Gaynor JW. Evaluation of failure to rescue as a quality metric in pediatric heart surgery: an analysis of the STS Congenital Heart Surgery Database. Ann Thorac Surg. 2012;94:573-9.

26. van Gameren M, Putnam LM, Takkenberg JJ, Bogers AJ. Risk stratification for adult congenital heart surgery. Eur J Cardiothorac Surg. 2011;39:490-4. 